\title{
Mutations du travail et « nouvelles » formes de discriminations : introduction
}

\section{DJaouidah Sehili et Tanguy Dufournet}

Djaouidah Sehili est professeure des universités à l'INSPE de Reims Champagne-Ardenne et chercheuse au CEREP - Centre d'Etudes et de Recherches sur les Emplois et les Professionnalisations. Tanguy Dufournet est doctorant au centre Max Weber (Université Lumière Lyon 2) où il prépare une thèse intitulée « Le travail pornographique gay : Jouissance et Aliénation - une sociologie située du travail (homo-)sexuel des corps ».

djaouidah.sehili@univ-reims.fr

tanguy.dufournet@univ-lyon2.fr

S'il est un domaine qui ne peut échapper aux inégalités de traitement et à toutes les formes de discrimination, c'est bien celui du travail. Or depuis les 50 dernières années, celui-ci subit des mutations profondes ${ }^{1}$. Pour le dire synthétiquement, la mise en œuvre de modalités de travail «flexibles » a impacté les conditions d'emploi et les rapports sociaux qui en découlent ${ }^{2}$. En effet, pour l'essentiel, ces «nouvelles » organisations combinent un usage massif des outils d'automation dorénavant associés aux technologies de l'information et

${ }^{1}$ Dufournet T., Rozenblatt P et Séhili D. (2019), « The creeping advance of working from home », in Green European Journal, septembre.

${ }^{2}$ Séhili D. (2003), La castration sociale, coll. Le Présent Avenir, Éditions Syllepse. 
de la communication ${ }^{3}$ (NTIC), permettant de coordonner, dans le temps et l'espace, des organisations internalisées et externalisées ; un ajustement de la production, matérielle ou de service, à la demande fluctuante des marchés ; et enfin et surtout un ajustement modulable des effectifs statutaires et/ou précaires. Ces transitions en cours sur le marché du travail ${ }^{4}$ s'accompagnent de transformations institutionnelle, juridique, sociale et sociétale, dont il est encore difficile de mesurer toutes les conséquences sur les personnes.

En fait, de nombreuses études montrent que ces « formes particulières » de mise au travail sont surtout un prélude au précariat ${ }^{5}$. En effet, alors que le contrat salarial s'était mu en statut, la crise de l'emploi et la mondialisation des échanges ont accentué les écarts à la «norme salariale » et les processus de précarisation. Également dites « atypiques ", du fait qu'elles se distinguent du modèle dominant des années 50 à 70 (CDI, temps plein et unicité de l'employeur), ces formes complexes de mise au travail reconfigurent les rythmes de vie et fragilisent la législation sociale et les acquis sociaux en redéfinissant fondamentalement les collectifs de travail ${ }^{6}$, jusqu'alors principalement fondées sur les métiers et les professions ${ }^{7}$, et durablement situés notamment dans un même espace de production délimité, comme l'usine ou le bureau. Sur quel « commun » ces collectifs se constituent-ils ? À partir de quelles expériences partagées se fédèrent-ils ? En somme, ces mutations du travail tendent-elles à une segmentation accrue ou une atomisation des salarié. e. s ? Aussi,

3 Carey J. (2014), « Retour vers le futur: comment les technologies de communication pénètrent dans les foyers américains », Réseaux, "Les cultural studies », n 80, pp. 145157.

4 Dhume F. (2007), «De la discrimination du marché au marché de la discrimination », Mouvements, «Le gouvernement des enfants », vol. 49, pp. 128136 ; Marchal E. (2015), Les embarras des recruteurs : enquête sur le marché du travail, Éditions de l'EHESS, coll. Cas de figure.

5 Colombo E. et Rebughini P. (2015), « Potentialités de l'intersectionnalité : réflexions à partir d'une recherche sur la précarité du travail chez les jeunes italiens "), Interrogations, n 20.

${ }_{7}^{6}$ Gorz A. (1980), Adieux au prolétariat : Au-delà du socialisme, Galilée.

${ }^{7}$ Hughes E. C. (1996), Le regard sociologique : essais choisis, Éditions de l'EHESS, coll. Recherches d'histoire et de sciences sociales. 
si collectif il y a, alors comment ces « nouveaux collectifs » sont-ils nommés, valorisés, ou dévalorisés ? Comment ces mutations transforment-elles ou limitent-elles les conditions de mobilisations à visées revendicatrices ou émancipatrices? Que disent ces collectifs du rapport au travail contemporain qui s'instaure ? Permettent-ils de réinventer la scission entre travailleur.euse.s et non-travailleur.euse.s ?

De plus, les mutations du travail en cours combinent des noyaux de personnels, souvent sous statuts différents (CDI/CDD/Intérim/travail saisonnier), l'usage de personnels permanents, mais aussi de plus en plus, l'usage de services et personnes externalisés entérinant ainsi l'idée d'une ubérisation incontournable (voire souhaitable) du travail. De fait, cette invalorisation massive du travail conduit à la création de figures contemporaines de la précarité. Paradoxalement, nous pouvons aussi voir dans ces processus d'invalorisation du travail ${ }^{8}$ 1'occasion d'intégrer aux «marchés de l'emploi » des populations jusqu'alors exclues. Mais alors, à quel prix/coût s'intègrent-elles ? À quoi doivent-elles éventuellement renoncer comme droits, acquis sociaux, qualité des conditions de travail, etc. ?

Dès les années 70, ces processus de redistribution des tâches et des statuts vont donc servir de base à une reconfiguration discriminante des hiérarchies de travail, dont la forme la plus exemplaire sera l'expansion de la sous-traitance et de l'intérim à des catégories sociales qui bénéficiaient jusqu'alors d'un statut et qui seront désormais marginalisées ${ }^{9}$. Sans aucun doute, ces mutations du travail transforment la hiérarchisation des compétences ${ }^{10}$ et des savoirs professionnels $^{11}$, tout en permettant d'instaurer une dialectique entre intérieur et extérieur dont l'enjeu est l'externalisation du « sale

${ }^{8}$ Rozenblatt P. (2017), Razzia sur le travail : critique de l'invalorisation du travail au XXI ${ }^{e}$ siècle, Éditions Syllepse, coll. Sens dessus-dessous.

${ }^{9}$ Coriat B. (1981), Science, technique et capital, Éditions du Seuil.

${ }^{10}$ Séhili D. (2001), «Évaluation sous influences à EDF-GDF », in Maugeri S. (dir.), Délit de gestion, La Dispute.

${ }^{11}$ Flocco G. (2015), Des dominants très dominés : pourquoi les cadres acceptent leur servitude, Raison d'agir. 
boulot $»^{12}$. Sans compter, qu'elles vont également modifier la valeur attribuée aux qualifications au travail ${ }^{13}$. Quelle place occupe cette hiérarchie des qualifications dans la production de normes discriminantes ? En somme, quelles sont les compétences aujourd'hui valorisées ou dévalorisées et quelles conséquences cela a-t-il sur les personnes ? De plus, si la productivité et la performance deviennent potentiellement les paradigmes renouvelés du rapport au travail alors on peut supposer que cela conduit inexorablement une extension des temps du travail au détriment d'autres temps.

Ce numéro des Cahiers de la Lutte Contre les Discriminations s'évertue à répondre à ces nombreuses questions en s'intéressant particulièrement à ces transformations du travail, tant dans leurs dynamiques de changements non stabilisées dans un contexte de chômage de masse, que dans leurs conséquences discriminatoires sur les personnes selon leur genre, leur classe, leur race, leur état de santé, leur âge, etc. Ce qui nous invite à les appréhender à la fois dans leur dimension interdisciplinaire et internationale, notamment dans un contexte d'une mondialisation loin d'être régulée, mais aussi plus finement en donnant à voir comment le travail est producteur de ses propres normes et hiérarchies raciales, genrées, classistes, etc ${ }^{14}$. Dans ce sillage, les contributions présentées mettent en perspective une approche intersectionnelle ${ }^{15}$, même lorsqu'elles ne mobilisent pas la notion, rendant compte de la complexité des discriminations.

Dans son article intitulé «Itinéraires instables et exposition aux risques discriminatoires », Emmanuelle Marchal aborde la question du

\footnotetext{
12 Hughes E. C. (1996), Le regard sociologique : essais choisis, Éditions de l'EHESS, coll. Recherches d'histoire et de sciences sociales.

13 Naville P. (2012), Essai sur la qualification du travail, Rééd. Rozenblatt P. et Sehili D. (dir.), Éditions Syllepse, coll. Sens dessus-dessous.

14 Séhili D. (2020), Pour une sociologie intersectionnelle du travail, Éditions Syllepse, coll. Présent Avenir, à paraître.

${ }^{15}$ Fassin É. (2015), « Les langages de l'intersectionnalité », Raisons politiques, «Les langages de l'intersectionnalité », vol. 3, n58, pp. 5- 7 ; Fassa F. et Lépinard É. (2016), L'intersectionnalité : enjeux théoriques et politiques, La Dispute, coll. Le genre du monde, 2016.
} 
lien entre l'instabilité des parcours professionnels des individus et les risques discriminatoires. L'enchaînement des contrats courts, leur nonrenouvellement, la volonté d'accéder à des emplois stables et à des emplois de titulaires, se traduisent, selon l'auteure, par des remises à l'épreuve périodiques des individu. e. s, alors même que les employeurs les connaissent déjà. Elles sont autant d'occasions d'opérer des jugements à caractère discriminatoires. Emmanuelle Marchal aborde cette question à partir de l'analyse des réclamations déposées au Défenseur des droits, concernant des embauches dans les secteurs public ou privé. Prenant appui sur une série d'études de cas, elle met particulièrement bien en évidence ce qui contribue à augmenter les attentes des réclamants et à déstabiliser les plaintes pour discrimination. Par ailleurs, la diversité des critères incriminés est ici soulignée d'autant qu'ils ne se limitent pas aux critères du sexe, de l'origine présumée, de la race ou de l'âge mais s'étendent à ceux de la santé, des grossesses, du handicap ou encore de l'appartenance syndicale.

Suivant la même logique, l'article de Maël Dif-Pradalier, au titre évocateur de «Changer la donne ou donner le change ? Enjeux et usages d'un groupement d'employeurs d'insertion et de qualification pour la grande entreprise » démontre bien la portée et les limites d'un groupement d'employeurs d'insertion et de qualification (GEIQ) qui, en tant qu'intermédiaire de l'emploi original et encore peu diffusé en France, est chargé par certaines entreprises de faire rentrer en son sein un public qui lui est d'ordinaire étranger (femmes, jeunes notamment issu. e. s de l'immigration et « séniors »). Le recours au GEIQ permet, selon l'auteur, à la grande entreprise donneuse d'ordre de résoudre des problèmes de recrutement sur des métiers « en tension » tout en contribuant à atteindre des objectifs en termes de politique de diversité et de RSE. Mais si des bénéfices sont bien réels pour les personnes sélectionnées sur la base de leur capacité à réaliser concrètement les tâches demandées et à tenir leur place, les effectifs concernés sont toutefois modestes. Et surtout, les mécanismes internes producteurs de hiérarchisation et de segmentation sociales des publics et des statuts d'emploi restent in fine inchangés. 
Ciblant les «Discriminations acceptables et le racisme inavouable dans le travail intérimaire », Grégory Giraudo-Baujeu, quant à lui, nous propose d'analyser les insertions professionnelles des salariés intérimaires, à travers l'observation de l'organisation du travail et des tâches dans les entreprises, ainsi que leurs relations avec les salarié. e. s permanent. e. s. Pour l'auteur, le statut d'emploi semble encore être aujourd'hui un critère de discrimination, notamment dans l'attribution du sale boulot. Non seulement ces discriminations sont acceptées et considérées comme acceptables par l'ensemble des acteurs de l'intérim - agences, entreprises, salariés intérimaires -, mais elles apparaissent, dans certains cas, comme des moyens confortables et sereins d'expression de formes de racisme. Derrière la hiérarchisation des postes assumée, se dessinerait une ethnicisation des tâches plus controversées. Derrière des discriminations acceptables, s'exprimerait un racisme inavouable.

C'est également de discriminations raciales dont il est question dans l'article d'Ibrahim Diallo intitulé « Discrimination raciale ou distinction en fonction de l'expérience professionnelle ? Des ambiguïtés qui posent question ». À partir de la démission d'un groupe de saisonniers subsahariens, l'auteur analyse la complexité des discriminations dans les grandes exploitations agricoles en Bretagne saisies a priori à l'aune de critères objectifs de gestion, comme ceux de l'expérience ou de l'ancienneté, et à ceux plus subjectifs des compétences et/ou savoirs perçus au travers de notions de fidélité ou de loyauté. Dans le discours des employeurs, ces critères expliqueraient la différence de traitement entre «nouveaux venus » et «anciens ». Toutefois, reste que cette discrimination (par l'expérience) est de toute évidence doublée d'une discrimination raciale, comme en témoigne le fait que les saisonniers qui se retrouvent systématiquement à la marge sont exclusivement des subsahariens.

Pour conclure ce numéro, c'est un article sous forme de plaidoirie que nous propose Patrick Rozenblatt. Son texte intitulé «Mutations du 
travail et renommées : quand les catégories de la statistique d'État discriminent les retraité. e. s en les classant comme "inactifs.ves" " entend exclusivement repenser les catégorisations de l'activité intégrant la réalité des formes de coopération propres à la division sociale du travail productif. Selon l'auteur, si les catégories statistiques de l'État servent théoriquement à mesurer des représentations du réel pour permettre l'élaboration de « justes » politiques publiques, il convient, toutefois, d'en déconstruire synthétiquement les catégories. Toutefois, il convient d'en déconstruire synthétiquement les catégories. Selon Patrick Rozenblatt, dorénavant, et avec l'expansion de processus d'invalorisation du travail, elles deviennent inégales et discriminantes : inégales parce qu'elles camouflent la réalité des rapports hiérarchiques de production et contribuent à rendre invisible la contribution d'une grande partie de la population à la production de valeur ; discriminatoires parce qu'elles permettent de montrer du doigt tels des « oisifs.ves » ou des « frelons » les inactifs.ves-retraité.e.s.

Dans ce numéro, l'ensemble des articles donne donc à voir un processus de détérioration du salariat induisant des formes spécifiques d'inégalités et de discriminations dont les enjeux de sociabilité qui en résultent sont loin d'être connus dans leurs conséquences et encore moins maîtrisés. Toutefois, même si cette approche des « discriminations au travail » peut paraître « classique » pour une lectrice ou un lecteur averti. e, c'est également des pistes qui sont ouvertes pour penser la production spécifique de ces « autres » formes de discrimination par et dans le travail. De ce point de vue, ce numéro et les contributions qu'il regroupe adoptent une perspective originale et encore peu documenté en France ${ }^{16}$, contrairement aux États-Unis où ce mouvement a été initié par Evangelina Holvino dans son article de 2008 «Intersections : The Simultaneity of Race, Gender and Class in Organization Studies ». En effet, pour elle, « reconceptualiser les intersections de la race, du genre et de la classe [...] peut appuyer

\footnotetext{
${ }^{16}$ Séhili D. (2020), Pour une sociologie intersectionnelle du travail, Éditions Syllepse, coll. Présent Avenir, à paraître.
} 
l'élaboration de nouvelles théories, recherches et pratiques dans les études sur les organisations » et montrer de quelles «façons les relations et la stratification de la race, du sexe et de la classe sont intégrées dans les structures organisationnelles, les processus et les méthodes de travail, qui semblent normales alors qu'elles produisent et reproduisent, en même temps, des relations particulières d'inégalités et de privilèges ${ }^{17}$. C'est là, notre ambition.

\section{Bibliographie :}

Carey J. (2014), « Retour vers le futur : comment les technologies de communication pénètrent dans les foyers américains », Réseaux, «Les cultural studies », $\mathrm{n}^{\mathrm{o}} 80$, pp. 145- 157.

Colombo E. et Rebughini P. (2015), « Potentialités de l'intersectionnalité : réflexions à partir d'une recherche sur la précarité $\mathrm{du}$ travail chez les jeunes italiens », Interrogations, « Penser l'intersectionnalitén », $\mathrm{n}^{\circ} 20$.

Coriat B. (1981), Science, technique et capital, Éditions du Seuil, 186 p.

Dhume F. (2007), « De la discrimination du marché au marché de la discrimination », Mouvements, « Le gouvernement des enfants », vol. 49, pp. 128- 136.

Dubar C. (2010), La crise des identités. L'interprétation d'une mutation, 4 éd., PUF, coll. Le lien social, 228 p.

Dufournet T., Rozenblatt P et Séhili D. (2019), «The creeping advance of working from home », in Green European Journal, septembre.

${ }^{17}$ Holvino E. (2008), « Intersections : The Simultaneity of Race, Gender and Class in Organization Studies », Gender, Work \& Organization, vol. 17, n 3, pp. 248- 277 
Fassa F. et Lépinard É. (2016), L'intersectionnalité : enjeux théoriques et politiques, La Dispute, coll. Le genre du monde, 282 p.

Fassin É. (2015), « Les langages de l'intersectionnalité », Raisons politiques, « Les langages de l'intersectionnalité », $\mathrm{n}^{\mathrm{o}} 58$, pp. 5- 7.

Flocco G. (2015), Des dominants très dominés : pourquoi les cadres acceptent leur servitude, Raison d'agir, $171 \mathrm{p}$.

Gorz A. (1980), Adieux au prolétariat : Au-delà du socialisme, Galilée, 240 p.

Holvino E. (2008), «Intersections : The Simultaneity of Race, Gender and Class in Organization Studies », Gender, Work \& Organization, vol. $17, n^{\circ} 3$, pp. $248-277$

Hughes E. C. (1996), Le regard sociologique : essais choisis, Éditions de l'EHESS, Recherches d'histoire et de sciences sociales, ${ }^{\circ}$ 70, 344 p.

Marchal E. (2015), Les embarras des recruteurs : enquête sur le marché du travail, Éditions de l'EHESS, coll. Cas de figure, $271 \mathrm{p}$.

Rozenblatt P. (2017), Razzia sur le travail: critique de l'invalorisation du travail au XXI siècle, Éditions Syllepse, coll. Sens dessus-dessous, $158 \mathrm{p}$.

Rozenblatt P. et Séhili D. (dir.) et Naville P. (2012), Essai sur la qualification du travail, Éditions Syllepse, coll. Sens dessus-dessous, $178 \mathrm{p}$.

Séhili D. (202), Pour une sociologie intersectionnelle du travail, Éditions Syllepse, coll. Présent Avenir, à paraître. 
«ALORS QUE LE CONTRAT SALARIAL

S'ÉTAIT MU EN STATUT, LA CRISE DE

L'EMPLOI ET LA MONDIALISATION DES ÉCHANGES ONT ACCENTUÉ LES ÉCARTS À

LA « NORME SALARIALE » ET LES PROCESSUS DE PRÉCARISATION. [...] CES MUTATIONS DU TRAVAIL TENDENT-ELLES

À UNE SEGMENTATION ACCRUE OU UNE ATOMISATION DES SALARIÉ.E.S ? AUSSI, COMMENT CES MUTATIONS

TRANSFORMENT-ELLES OU LIMITENTELLES LES CONDITIONS DE MOBILISATIONS

À VISÉES REVENDICATRICES OU ÉMANCIPATRICES ? QUE DISENT CES COLLECTIFS DU RAPPORT AU TRAVAIL CONTEMPORAIN QUI S'INSTAURE? PERMETTENT-ILS DE RÉINVENTER LA SCISSION ENTRE TRAVAILLEUR.EUSE.S ET NON-TRAVAILLEUR.EUSE.S ? »

\section{Djaouidah Sehili \& Tanguy Dufournet}

